psychopraxis. neuropraxis $2017 \cdot 20: 227$ https://doi.org/10.1007/s00739-017-0438-y

Online publiziert: 13. November 2017

(c) Springer-Verlag GmbH Austria 2017

\section{Nilufar Mossaheb}

Universitätsklinik für Psychiatrie und Psychotherapie, Klinische Abteilung für Sozialpsychiatrie,

Medizinische Universität Wien, Wien, Österreich

\title{
Tatsächlich ist vieles möglich!
}

Liebe Leserinnen, liebe Leser,

Sie halten in Ihren Händen die aktuelle Ausgabe der Zeitschrift psychopraxis.neuropraxis, die die Themen psychiatrische Versorgung, Alkoholabhängigkeit, Parkinson-Psychosen sowie Kognition bei multipler Sklerose anhand spannender Kasuistiken darstellt.

Die Gewährleistung einer vielfältigen psychiatrischen Versorgungslandschaft ist für eine bedarfsorientierte und individualisierte Behandlung von Menschen mit psychiatrischen Erkrankungen essenziell. Das bedeutet zum Beispiel, dass ein niederschwelliger Zugang zu entsprechend früher Diagnostik und adäquaten medikamentösen als auch psychotherapeutischen Behandlungen möglich ist. Das bedeutet aber auch, dass Behandlungen strukturell auf die Bedürfnisse des Individuums angepasst werden können, indem sie stationär, teilstationär, ambulant, über Besuche am Wohnort oder über ein Fallmanagement-Team angeboten werden.

In diesem Heft finden Sie einige Beispiele von spezialisierten tagesklinischen Einrichtungen, die dem Bedarf an individualisierter, alltagsangepasster und ressourcenorientierter Behandlung auf höchstem Niveau gerecht werden. Häufig sind es die Übergänge, die besonderer Unterstützung bedürfen, etwa vom stationären in den ambulanten Bereich, vom Krankenstand über die Rehabilitation in eine Reintegration ins Arbeitsleben oder in die Ausbildung, von der Ohnmacht des Erkrankens zur Selbstwirksamkeit konstruktiver Strategien sowie von der Adoleszenz ins Erwachsenenalter (Stichwort Transitionspsychiatrie). Sowohl klinisch als auch wissenschaftlich wird weltweit an Präventions- und Transitionsstrategien gearbeitet. Tatsächlich ist dies auf vielen Ebenen möglich!

In diesem Sinne ist die Gewährleistung einer flächendeckenden hochqualitativen allgemeinpsychiatrischen Versorgung als wesentlich zu sehen; jedoch ebenso der weitere Ausbau von spezifischen psychiatrischen Einrichtungen mit einem integrierten Ansatz, der dem Individuum und dem Anspruch an dessen optimaler Diagnostik und Behandlung gerecht wird.

Ich wünsche Ihnen eine interessante Lektüre!

Ihre

Nilufar Mossaheb

\section{Korrespondenzadresse}

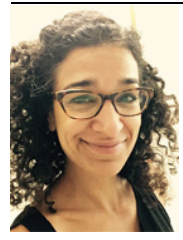

Dr. N. Mossaheb, M.Sc. Universitätsklinik für Psychiatrie und Psychotherapie, Klinische Abteilung für Sozialpsychiatrie, Medizinische Universität Wien Währinger Gürtel 18-20, 1090 Wien, Österreich nilufar.mossaheb@ meduniwien.ac.at

Interessenkonflikt. N. Mossaheb gibt an, dass kein Interessenkonflikt besteht. 